\title{
EDITORIAL AND COMMENT \\ The Healthcare Safety-Net: Time for Greater Transparency and Accountability?
}

\author{
Michael Hochman, MD, MPH
}

University of Southern California, Los Angeles, CA, USA.

J Gen Intern Med 35(4):983-4

DOI: $10.1007 / \mathrm{s} 11606-020-05667-8$

(c) Society of General Internal Medicine 2020

$\mathrm{D}$ ue to resource limitations and an ever-growing population of vulnerable patients, policy makers frequently worry about the quality of care within safety-net health systems. The results of an analysis by Oronce and Fortuna ${ }^{1}$ in this month's issue of JGIM may provide at least some reassurance.

In the study, the authors compare quality metrics between U.S. community health centers - safety-net clinics that primarily care for low-income and vulnerable populations - with those of private primary care practices. Using data from the National Ambulatory Medical Care Survey (NAMCS), the authors examined 12 measures of "highvalue" care along with 7 measures of "low-value" care. The results showed that community health centers performed as well, if not better, on virtually every metric compared with private practices. For example, clinicians at community health centers were more likely than private practice clinicians to appropriately provide beta blockers for patients with congestive heart failure and less likely to deliver inappropriate screening EKGs and urinalyses during general medical exams. These results provide important evidence that some of the most vulnerable members of our society generally receive technically sound care.

As a primary care doctor who previously served as a medical director at a community health center, I find myself relieved by these findings - which, despite common anecdotal reports to the contrary, are consistent with prior studies. ${ }^{2}$ But there are substantial caveats.

First, it is important to recognize important quality gaps in private U.S. health systems, to which the community health centers were compared. Overall, U.S. patients only receive appropriate, necessary care half to three-quarters of the time. ${ }^{3}$ Thus, the fact that community health centers achieve comparable - or somewhat better-technical quality scores compared with private practices may not be cause for too much celebration.

Second, technical quality represents just one measure of health system performance. Evidence suggests that the U.S. safety-net systems suffer from other important quality

Published online January 21, 2020 shortcomings. In particular, safety-net institutions often perform sub-optimally with respect to customer service, telephone, and patient portal responsiveness and appointment scheduling ${ }^{4}$ - all factors that may impact health outcomes. I suspect that a study examining patient experience measures would not be as favorable to community health centers as the results of this analysis by Oronce and Fortuna.

Another reason why this study's findings may only tell part of the story is that, as the authors highlight, the results may not be applicable to "clinical quality measures that might be more susceptible to patients' social determinants of health, such as ambulatory care-sensitive admissions and all-cause 30-day readmissions." 1 Other measures that are more influenced by social determinants, such as acute care utilization rates, chronic disease outcomes, and overall health might demonstrate important gaps between community health center and private practice settings. ${ }^{5}$ Indeed, patients in the safety-net suffer from alarming health disparities, ${ }^{6}$ suggesting that there are other key factors that influence clinical outcomes besides technical quality.

Finally, it is important to note that this analysis only applies to community health centers, not to other types of safety-net institutions, such as public clinics, private safety-net groups that are not federally qualified health centers, the Indian Health Service, or the VA Health System.

Despite these caveats, the finding that community health centers deliver care of similar or better technical quality compared with private practices is noteworthy. These results may alter long-held and incorrect beliefs about deficiencies in care delivered by community health centers.

The new findings also have important policy implications. In recent years, commercial and Medicare groups have increasingly faced public scrutiny regarding their quality scores (e.g., Medicare's Physician Compare tool and analogous programs for commercial groups). However, safety-net providers have largely escaped this examination. ${ }^{7}$ In fact, safety-net administrators have, at times, resisted attempts to promote transparency, citing a lack of resources and infrastructure as well as concerns that closer quality monitoring might drive providers to leave safety-net care. ${ }^{8}$ The study by Oronce and Fortuna should encourage these administrators to be more open to external evaluation of quality. Already some regions of the country-such as 
Massachusetts - are beginning to demand greater transparency from their safety-net providers. ${ }^{9}$

The new findings also suggest that the safety-net may be ready for greater accountability in the form of value-based payment models. Thus far, community health centers, as well as other safety-net leaders, have been cautious about embracing alternatives to fee-for-service, such as capitation and risk-bearing contracts, citing concerns that such models might comprise already vulnerable safety-net funding streams. ${ }^{10}$ Oronce and Fortuna's findings demonstrate that, at least from the standpoint of technical quality, safety-net institutions appear to perform as well (or better) than their private counterparts. This ought to prompt more serious consideration of value-based models, as long as compensation is equitable for the challenges of the population served.

Overall, this study by Oronce and Fortuna provides provocative and exciting data, suggesting that pre-conceived notions about quality in the safety-net may be inaccurate. It also highlights the need for further research examining a broad array of safety-net quality metrics, including patient experience and health outcomes.

In the meantime, these findings should stimulate a renewed push for transparency and accountability in the safety-net. The public deserves the same level of oversight and expectations for the safety-net as for other provider groups. Thanks to the findings by Oronce and Fortuna, as well as other similar results, the argument that safety-net institutions lack the infrastructure to provide technically proficient care is increasingly losing its buoyancy.

Acknowledgments: I would like to thank Barbara J Turner, MD, MSEd, and Sonali Saluja, MD, MPH, for reviewing earlier versions of this manuscript.
Corresponding Author: Michael Hochman, MD, MPH; University of Southern California, Los Angeles, CA, USA (e-mail: Michael. Hochman@med.usc.edu).

\section{REFERENCES}

1. Oronce CIA, Fortuna RJ. Differences in rates of high-value and lowvalue care between community health centers and private practices. J Gen Intern Med. 2019. https://doi.org/10.1007/s11606-019-05544-Z

2. Hicks LS, et al. The quality of chronic disease care in US community health centers. Health Affairs 25.6 (2006): 1712-1723.

3. Levine DM, Linder JA, Landon BE. The quality of outpatient care delivered to adults in the United States, 2002 to 2013. JAMA Intern Med. 2016;176(12): 1778-1790.

4. Katz MH. Future of the safety net under health reform. Jama 2010;304(6): 679-680.

5. Raphael JL, et al. The medical home experience of low-income children with non-urgent encounters in acute care settings. J Health Care Poor Underserved. 2016;27(4): 1885-1898.

6. Internet Citation: 2018 National Healthcare Quality and Disparities Report. Content last reviewed September 2019. Agency for Healthcare Research and Quality, Rockville, MD. https://www.ahrq.gov/research/ findings/nhqrdr/nhqdr18/index.html

7. "Medicaid Providers Operating Under the Radar, Health Affairs Blog, December 18, 2018. https://doi.org/10.1377/hblog20181217.506070

8. See https://www.centerforhealthjournalism.org/2018/07/10/free-datapublic-accountability-sorely-needed-medicaid-providers

9. See: https://www.centerforhealthjournalism.org/2018/08/09/massachusetts-pioneers-making-quality-public-priority-safety-net-patients

10. Bhatia J, Tobey $\mathbf{R}$, Hochman $\mathbf{M}$. Value-based payment models for community health centers: time to (cautiously) take the plunge? JAMA. 2017;317(22):2275-2276. https://doi.org/10.1001/jama.2017.5174

Publisher's Note Springer Nature remains neutral with regard to jurisdictional claims in published maps and institutional affiliations. 